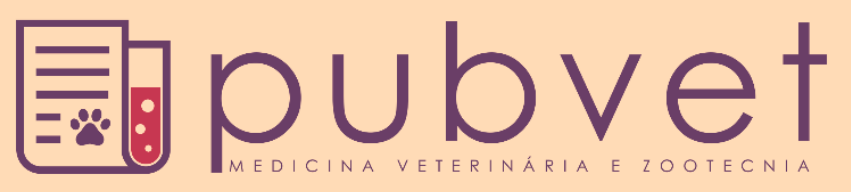

https://doi.org/10.31533/pubvet.v16n01a1003.1-7

\title{
Efeito do uso de óleos essenciais no desempenho de bovinos de corte confinados
}

\author{
Guilherme Lobato Menezes $^{10}$, Alan Figueiredo de Oliveira $^{1 \bullet}$, Pamella Grossi de Sousa $^{1}$, $^{\circ}$ \\ Frederico Patrus Ananias de Assis Pires ${ }^{1}{ }^{\circ}$, Rafael Araújo de Menezes ${ }^{1}{ }^{\circ}$, Eduarda Chaves de \\ Oliveira $^{2 * \bullet}$, Ana Eliza da Silva ${ }^{2}$, Lúcio Carlos Gonçalves ${ }^{3}{ }^{\circledR}$, Diogo Gonzaga Jayme ${ }^{30}$ \\ ${ }^{I}$ Doutorando em Zootecnia, Escola de Veterinária da Universidade Federal de Minas Gerais. Belo Horizonte - MG, Brasil \\ ${ }^{2}$ Discente de Medicina Veterinária -Pontifícia Universidade Católica de Minas Gerais. Betim-MG, Brasil \\ ${ }^{3}$ Prof. Departamento de Zootecnia, Escola de Veterinária da Universidade Federal de Minas Gerais. Belo Horizonte-MG, Brasil. \\ *Autor para correspondência. E-mail: eduardahchaves@gmail.com
}

Resumo. Ionóforos são compostos amplamente utilizados nas composições de dietas de ruminantes, com o objetivo de diminuir o consumo de matéria seca e aumentar a eficiência alimentar, dificultando o desenvolvimento da acidose ruminal e gerando melhor aproveitamento de nutrientes, por ação principalmente sobre as bactérias gram-positivas. Entretanto, essa utilização é uma preocupação pelo surgimento de resistência bacteriana aos antibióticos, que reflete na medicina humana. Meio a situação, surgem estudos com os óleos essenciais (OEs) que buscam a substituição dos antibióticos, promovendo efeitos similares, devido suas propriedades antimicrobianas, similares às funções dos ionóforos. $\mathrm{O}$ objetivo do trabalho foi analisar os resultados da comparação entre os dois aditivos na dieta. Os efeitos dos OEs variam de acordo com sua fonte, podendo causar diferentes impactos no consumo de alimentos pelo animal; porém, estudos realizados concluíram que independente da dosagem e da fonte os óleos provocam resultado positivo no ganho médio diário (GMD), levando a eficiência alimentar (EA) a não sofrer impactos pelo aumento do consumo de matéria seca (CMS). Colocando-se os OEs em substituição à monensina, a observação do aumento do CMS se mantém; porém, não são observadas alterações no GMD, também é notado um aumento na incidência de abcessos hepáticos que gera a necessidade de novos estudos para compreensão. $\mathrm{O}$ fornecimento dos OEs na alimentação terá repercussão similar a monensina (principal ionóforo utilizado); porém, com um maior CMS.

Palavras-chave: Confinamento, antibióticos ionóforos, resistência

\section{Effect of the use of essential oils on the performance of confined beef cattle}

Abstract. Ionophores are compounds widely used in the composition of ruminant diets, with the objective of reducing dry matter intake and increasing feed efficiency, hindering the development of ruminal acidosis and generating better use of nutrients, mainly by acting on gram-positive bacteria. However, this use is a concern due to the emergence of bacterial resistance to antibiotics, which is reflected in human medicine. Amid the situation, there are studies with essential oils (EOs) that seek the replacement of antibiotics, promoting similar effects, due to their antimicrobial properties, similar to the functions of ionophores. The objective of this work was to analyze the results of the comparison between the two additives in the diet. The effect of EOs varies according to their source, and may cause different impacts on the animal's food consumption, but studies concluded that regardless of dosage and source, oils cause a positive result in average daily gain (GMD), leading to feed efficiency (EA) not to be impacted by the increased consumption of dry matter (DMC). Putting EOs in place of monensin, the observation of the increase in CMS is maintained, but no changes are observed in the GMD, an increase in the incidence of liver 
abscesses is also noted, which creates the need for further studies for understanding. The supply of EOs in food will have repercussions similar to monensin (the main ionophore used), but with a higher CMS.

Key words: Feed-lot, ionophore antibiotics, resistance

\section{Introdução}

Os sistemas de confinamento aumentaram a inclusão de alimentos concentrados nas dietas nos últimos anos (Monteschio et al., 2017; Oliveira \& Millen, 2014; Pinto \& Millen, 2018). Esse aumento pode ocasionar problemas metabólicos, como as acidoses e as laminites, que juntas representam 37,9\% das causas de adoecimento nesse sistema (Abdela, 2016; Nagaraja \& Titgemeyer, 2007; Pinto \& Millen, 2018). As estratégias nutricionais são importantes para manter a saúde e potencializar o desempenho dos animais. Uma dessas estratégias é a utilização de ionóforos com ação antibiótica que são comumente adicionados às dietas para aumentar a concentração de ácido propiônico no rúmen melhorando a eficiência energética e o desempenho animal (Chaucheyras-Durand \& Ossa, 2014; Henderson et al., 2015; Matthews et al., 2019; Myer et al., 2015). Os ionóforos, além de possibilitar melhor desempenho, podem reduzir os riscos de acidose e por isso são amplamente utilizados (Torres et al., 2021).

Nos últimos anos cresceu a preocupação em relação ao uso de antibióticos na alimentação animal devido ao aparecimento de bactérias resistentes que podem causar riscos à saúde humana (Schäberle \& Hack, 2014). Um estudo realizado por Manero et al. (2006) demonstrou semelhança entre os isolados de enterococos resistentes à vancomicina e eritromicina em esgotos da área urbana e nos dejetos de suínos, sugerindo que cepas resistentes aos microrganismos circularam das fazendas para as cidades. Em meio a um cenário crescente de preocupações, os óleos essenciais (OEs) surgem como uma medida alternativa na nutrição animal para substituir antibióticos e ionóforos (Rivaroli, 2013; Rivaroli et al., 2016, 2017). Esses são compostos aromáticos extraídos de várias partes de plantas incluindo folhas, raízes, caule, flores e casca (Patra, 2010). Os OEs possuem ação semelhante aos ionóforos com ação antibiótica e podem atuar na manipulação da fermentação ruminal e no controle de doenças metabólicas (Benchaar et al., 2003; Torres et al., 2021). Assim, objetivou-se revisar a influência de uso de OEs no desempenho de bovinos de corte confinados e os impactos na saúde e no desempenho da substituição de antibióticos ionóforos por OEs.

\section{Atividade dos óleos essenciais}

Os OEs são metabólitos secundários formados por plantas aromáticas extraídos geralmente por vapor ou hidro destilação e são compostos aromáticos voláteis que possuem forte odor e natureza hidrofóbica com alta afinidade pelos lipídios da membrana celular (Benchaar et al., 2008). Quimicamente, os OEs são formados por misturas variáveis de terpenóides, principalmente os monoterpenos (C10) e os sesquiterpeno (C15). Entretanto, diterpenos (C20) podem ser encontrados, além de hidrocarbonetos alifáticos de baixo peso molecular (lineares, ramificados, saturados e insaturados), ácidos, álcoois, aldeídos, lactonas e, excepcionalmente, compostos contendo nitrogênio, enxofre, cumarinas e homólogos de fenilpropanoides (Dorman \& Deans, 2000).

Os OEs por possuírem afinidade com os lipídios de membrana têm demonstrado propriedades antimicrobianas, incluindo bactérias, protozoários e fungos (Greathead, 2003). Na nutrição de ruminantes confinados os principais benefícios são as melhorias de desempenho e a redução das doenças metabólicas ocasionadas pela alta inclusão de concentrado como as acidoses láticas, laminites, paraqueratoses e os abscessos hepáticos. Existem evidências que algumas misturas de OEs contendo limoneno, eugenol, vanilina, guaiacol e timol podem reduzir a incidência de abscessos hepáticos em animais consumindo grandes quantidades de concentrados (Meyer et al., 2009; Torres et al., 2021) possivelmente pela redução no crescimento de bactérias da espécie Fusobacterium necrophorum.

As bactérias gram-positivas são mais sensíveis à ação dos OEs que as gram-negativas (Burt, 2004). Esse efeito é semelhante ao exercido pela monensina (Marshall \& Levy, 2011; Matos et al., 2021), o que reduz os riscos de acidose ocasionados pela alta proporção de concentrado na dieta e potencializa o desempenho animal. Os OEs podem possuir concentrações diferentes e geralmente dois ou três componentes representam de 20 a 70\% das propriedades biológicas dos OEs (Bakkali et al., 2008). O 
efeito dos OEs pode depender da fonte e da dose, assim é necessário ter cautela em generalizar os resultados sobre a utilização destes.

\section{Efeito dos OEs no desempenho animal}

Fugita et al. (2018) avaliaram novilhos alimentados sem OEs, com a adição de 4 g/animal/dia de OEs de mamona e caju (rico em ácidos ricinoléico, anacárdico, cardol e cardanol) ou com adição de 4 g/animal/dia de uma mistura de OEs de orégano (rico em carvacrol), mamona e caju. As dietas foram isoenergéticas e isonitrogenadas com 50\% de concentrado. A inclusão dos OEs não alterou $(\mathrm{P}>0,05) \mathrm{o}$ consumo de matéria seca (CMS) e a eficiência alimentar (EA), mas a inclusão de OEs de orégano, mamona e caju aumentaram ( $\mathrm{P}<0,01)$ o ganho de peso em $14,7 \%$, o peso final em $5,54 \%$ e o peso de carcaça em 5,37\% quando comparado aos animais não alimentados com OEs. Esses resultados demonstram a melhoria no desempenho a partir da inclusão de OEs mesmo em dietas com menor inclusão de concentrado, podendo ser uma boa alternativa em dietas de adaptação por não reduzir o consumo.

Em dietas com $90 \%$ de concentrado, Ornaghi et al. (2017) avaliaram o uso de OEs extraídos da folha de cravo nas concentrações de 3,5 g/animal/dia (CLO35 - contendo 370, 58 e 5,69 mg/kg de MS de eugenol, carofilenina e acetato de eugenila, respectivamente) ou $7 \mathrm{~g} / \mathrm{animal} / \mathrm{dia}$ (CLO70 - contendo 740, 116 e $11,38 \mathrm{mg} / \mathrm{kg}$ de MS de eugenol, carofilenina e acetato de eugenila, respectivamente) e OEs extraídos da folha de canela nas concentrações de 3,5 g/animal/dia (CIN35 - contendo 350, 21 e 14,35 $\mathrm{mg} / \mathrm{kg}$ de MS de cinamaldeído, carofileno e alfa-pineno, respectivamente) ou $7 \mathrm{~g} / \mathrm{animal} / \mathrm{dia}$ (CIN70 contendo 700, 42 e 28,7 mg/kg de MS de cinamaldeído, carofileno e alfa-pineno, respectivamente). Os autores observaram maior $(\mathrm{P}<0,01)$ ganho médio diário (GMD) e peso final nos animais terminados utilizando OEs na dieta independente da fonte e dose. Em média os animais apresentaram aumentos de $14,7 \%$ no GMD $(1,47$ vs. 1,28) e de 8,6\% no peso final (494 vs. 455). Além disso, os animais alimentados com OEs apresentaram melhor $(\mathrm{P}<0,01)$ CMS (7,90 vs. 6,94). Entretanto, devido ao melhor desempenho, a EA não foi influenciada $(\mathrm{P}=0,23)(0,185$ vs. 0,180$)$ pelos tratamentos. Esses resultados demonstram que apesar dos OEs aumentarem o CMS, o melhor desempenho pode não influenciar a EA. $\mathrm{O}$ melhor desempenho pode reduzir os dias confinados que pode reduzir o custo operacional, aumentar o número de ciclos produtivos e a rentabilidade nas propriedades. Os autores ressaltaram que a inclusão de OEs nas dietas de bovinos dependerá da relação custo-benefício deste aditivo.

Souza et al. (2019) avaliaram o uso de óleos essenciais no desempenho de 40 novilhas Nelore. Os animais foram adaptados por sete dias, seguidos de 73 dias experimentais até atingirem $356 \pm 32,6 \mathrm{~kg}$. Foram avaliados cinco tratamentos: sem óleo essencial; com óleo essencial de alecrim (ROS) (4 g/animal/dia); uma mistura protegida de eugenol, timol e vanilina (BLE) (4 g/animal/dia); uma mistura protegida de eugenol, timol e vanilina (2 g/animal/dia) mais óleo essencial de cravo (BCL) (2 g/animal/dia); uma mistura protegida de eugenol, timol e vanilina (1,33 g/animal/ dia) mais óleo essencial de alecrim (1,33 g/animal/dia) mais óleo essencial de cravo (BRC) (1,33 g/animal/dia). Os animais que consumiram a mistura protegida de BLE, BCL e BRC não diferiram $(\mathrm{P}>0,05)$ quanto ao CMS, GMD e EA. Entretanto, apresentaram o CMS 20,7\% (6,12 vs. 5,07) maior $(\mathrm{P}=0,01)$ quando comparado ao grupo alimentado com ROS. O GMD aumentou $(\mathrm{P}=0,02) 102 \%(0,95$ vs. 0,47$)$ em relação aos animais alimentados com ROS e a EA melhorou $(\mathrm{p}=0,01)(0,156 ; 0,09)$ em $74,1 \%$. O CMS dos animais no grupo controle $(5,49)$ foi pior $(\mathrm{P}=0,01)$ que os animais no grupo BLE e BRC (em média $6,23)$ e não diferiu $(\mathrm{P}>0,05)$ do tratamento $\mathrm{BCL}(5,90)$. Entretanto, os animais do grupo controle apresentaram maior $(\mathrm{P}=0,02$; $\mathrm{GMD}(0,70$ vs. 0,47$)$ e melhor $(\mathrm{P}=0,01) \mathrm{EA}(0,13$ vs. 0,09$)$ que o grupo alimentado com ROS. Os óleos essenciais podem conter compostos voláteis que reduzem o CMS e a EA. O melhor resultado dos óleos essenciais protegidos provavelmente ocorreu pelo sinergismo das misturas que em $\mathrm{pH}$ apropriado $(5,5)$ melhorou a atividade das moléculas presentes em cada óleo essencial sem reduzir o CMS.

\section{Efeito da substituição de monensina por OEs no desempenho de bovinos confinados}

Pinto \& Millen (2018) demonstraram por meio de um questionário respondido por 33 nutricionistas, responsáveis pela formulação das dietas de aproximadamente $95 \%$ dos bovinos confinados no Brasil, que $100 \%$ dos confinamentos assessorados por eles utilizavam ionóforos. Nos EUA, Samuelson et al. (2016) demonstraram por meio de um estudo com questionário que, em média, 92,3\% dos nutricionistas 
entrevistados relataram fornecer ionóforos nas dietas de adaptação e 97,3\% nas dietas de terminação. Neste estudo foi demonstrado que o principal ionóforo utilizado para as fases de adaptação e terminação foi a monensina, representado por $77,3 \%$ e $100,0 \%$ das respostas respectivamente. Além de prevenir doenças metabólicas, muitos nutricionistas utilizam a monensina pelo potencial de maximização na EA, por reduzir o consumo sem reduzir o desempenho (Torres et al., 2021). Esse ionóforo por ser amplamente utilizado será o foco da discussão de substituição por OEs.

Meschiatti et al. (2019) avaliaram uma combinação de OEs e enzimas exógenas $\alpha$-amilase no desempenho de bovinos de corte confinados. Os animais iniciaram o confinamento consumindo $25 \%$ de bagaço de cana com retiradas graduais até atingir $8,5 \%$ do volumoso no $16^{\circ}$ dia de experimento. Os animais foram alimentados por 93 dias e foram avaliados com os seguintes tratamentos: monensina de sódio (26 mg/kg de MS); mistura de óleos essenciais (90 mg/kg MS); mistura de óleos essenciais + monensina (90 e $26 \mathrm{mg} / \mathrm{kg} \mathrm{MS}$, respectivamente); mistura de óleos essenciais $+\alpha$-amilase exógena (90 e $560 \mathrm{mg} / \mathrm{kg} \mathrm{MS}$, respectivamente). Os animais que consumiram OEs não diferiram (P > 0,05) quanto ao GMD (1,67 vs. 1,57), ao peso final (486 vs. 476) e ao peso de carcaça (272 vs. 265) em comparação com os que consumiram monensina. Os animais alimentados com OEs aumentaram ( $\mathrm{P}<0,001)$ o CMS (9,24 vs. 8,65 kg/dia) em 6,8\% comparado aos animais que consumiram monensina. Entretanto, a EA não diferiu entre os tratamentos. A combinação de $\mathrm{OEs}$ com monensina diminuiu $(\mathrm{P}<0,001)$ o CMS quando comparado ao OEs isoladamente $(8,5$ vs. 9,24) e não influenciou $(\mathrm{P}>0,05)$ o peso final, o peso de carcaça, o GMD e a EA quando comparado a monensina e OEs avaliados isoladamente. A combinação $\alpha$-amilase exógena aumentou o CMS (P < 0,001) em 9,36\% $(9,46$ vs. 8,65 kg/dia) quando comparado a monensina isoladamente, 2,38\% (9,46 vs. 9,24 kg/dia) quando comparado a mistura de óleos essenciais e 11,3\% quando comparado a mistura de óleos essenciais + monensina. Como consequência, os animais neste tratamento apresentaram maior peso final e $\mathrm{GMD}(\mathrm{P}<0,001$ e $\mathrm{P}=0,002)$ quando comparado com animais suplementados com monensina. Esses resultados demonstram que os OEs podem ser utilizados na substituição da monensina sem reduzir o ganho de peso e a EA. O uso da associação entre a monensina e o OEs não faz sentido no atual contexto que busca a redução do uso de ionóforos com ação antibiótica e a utilização de enzimas não difere das respostas promovidas pelo uso de OEs.

Toseti et al. (2020) também avaliaram em um estudo com arranjo fatorial 2 x 2 o desempenho de 88 novilhos Nelore terminados com duas fontes de volumoso (silagem de milho ou bagaço de cana-deaçúcar) combinados ao uso de monensina de sódio $(26 \mathrm{mg} / \mathrm{kg}$ de MS) ou a combinação de uma mistura de óleos essenciais (timol, eugenol, vanilina e limoneno) $+\alpha$-amilase exógena (90 e $560 \mathrm{mg}$ de produto comercial/kg MS, respectivamente). Os animais foram adaptados com inclusão crescente de concentrado por 21 dias e alimentados com dietas de terminação com alto concentrado (90\%) por 87 dias. Os animais alimentados com OEs apresentaram maior $(\mathrm{P}=0,023)$ peso final $(527$ vs. 510$)$ e de carcaça $(\mathrm{P}=0,029)(298 v s .288 \mathrm{~kg})$ ao término do estudo. Animais alimentados com a mistura de $\mathrm{OEs}$ também apresentaram aumento $(\mathrm{P}=0,043)$ de $8,51 \%$ no $\mathrm{GMD}(1,53$ vs. $1,41 \mathrm{~kg} / \mathrm{dia})$. Apesar dos efeitos da monensina na redução de CMS descritos na literatura, nesse estudo não foi observada diferença $(\mathrm{P}=$ $0,171)$ no $\mathrm{CMS}$ e na $\mathrm{EA}(\mathrm{P}=0,220)$.

Um estudo meta-analítico realizado por Torres et al. (2021) avaliou-se o uso de OEs em dietas como alternativa à monensina nas dietas para bovinos de corte confinados. Foram incluídos 10 estudos revisados por pares a partir de 262 encontrados pela estratégia de busca. Esses estudos demonstraram que o uso de OEs quando utilizado como substituto da monensina aumentam o CMS ( $\mathrm{P}<0,0001)$, não altera o peso final $(\mathrm{P}=0,536)$, o GMD $(\mathrm{P}=0,883)$, o peso de carcaça $(\mathrm{P}=0,085)$ e a $\mathrm{EA}(\mathrm{P}=0,106)$. Entretanto, o risco de abscesso hepático aumentou $(\mathrm{P}=0,036)$ com uso dos OEs. Contudo, é valido ressaltar que essa resposta veio de um pequeno número de comparações $(n=5)$ e que a estratégia de busca utilizada na revisão pode ter sido falha por ser feita em apenas uma base de dados, PubMed. Uma revisão sistemática do tema abrangendo maior número de bases de dados poderia resgatar mais estudos e apresentar resultado distintos quanto ao risco de abscessos hepáticos.

\section{Considerações finais}

Os OEs quando utilizados como aditivo podem aumentar o desempenho animal comparado aos animais não suplementados. A substituição de ionóforos ou antibióticos não reduziu o ganho de peso e a EA. A ocorrência de abscessos hepáticos precisa ser estudada por meio de uma revisão sistemática 
mais abrangente ou da condução de estudos primários. Por fim, os OEs podem ser uma boa alternativa para diminuir o uso de ionóforos e antibióticos na alimentação animal, o que tem sido uma preocupação crescente nos últimos anos.

\section{Referências bibliográficas}

Abdela, N. (2016). Sub-acute ruminal acidosis (SARA) and its consequence in dairy cattle: A review of past and recent research at global prospective. Achievements in the Life Sciences, 10(2), 187-196. https://doi.org/10.1016/j.als.2016.11.006.

Bakkali, F., Averbeck, S., Averbeck, D., \& Idaomar, M. (2008). Biological effects of essential oils-a review. Food and Chemical Toxicology, 46(2), 446-475.

Benchaar, C., Calsamiglia, S., Chaves, A. V, Fraser, G. R., Colombatto, D., McAllister, T. A., \& Beauchemin, K. A. (2008). A review of plant-derived essential oils in ruminant nutrition and production. Animal Feed Science and Technology, 145(1-4), 209-228. https://doi.org/10.1016/j.anifeedsci.2007.04.014.

Benchaar, C., Petit, H. V, Berthiaume, R., Ouellet, D. R., \& Chiquette, J. (2003). Effects of essential oil supplements on ruminal fermentation, rumen microbial populations and in sacco degradation of dry matter and nitrogen in the rumen of lactating dairy cows. Canadian Journal of Animal Science, $83,637$.

Burt, S. A. (2004). Essential oils: their antibacterial properties and potential applications in foods-a review. International Journal of Food Microbiology, 94(3), 223-253. https://doi.org/10.1016/j.ijfoodmicro.2004.03.022.

Chaucheyras-Durand, F., \& Ossa, F. (2014). The rumen microbiome: composition, abundance, diversity, and new investigative tools. The Professional Animal Scientist, 30(1), 1-12.

Dorman, H. J. D., \& Deans, S. G. (2000). Antimicrobial agents from plants: antibacterial activity of plant volatile oils. Journal of Applied Microbiology, 88(2), 308-316. https://doi.org/10.1046/j.1365-2672.2000.00969.x

Fugita, C. A., Prado, R. M., Valero, M. V., Bonafé, E. G., Carvalho, C. B., Guerrero, A., Sañundo, C., $\&$ Prado, I. N. (2018). Effect of the inclusion of natural additives on animal performance and meat quality of crossbred bulls (Angus vs. Nellore) finished in feedlot. Animal Production Science, 58(11), 2076-2083. https://doi.org/10.1071/AN16242.

Greathead, H. (2003). Plants and plant extracts for improving animal productivity. Proceedings of the Nutrition Society, 62(2), 279-290.

Henderson, G., Cox, F., Ganesh, S., Jonker, A., Young, W., Abecia, L., Angarita, E., Aravena, P., \& Arenas, G. N. (2015). Rumen microbial community composition varies with diet and host, but a core microbiome is found across a wide geographical range. Scientific Reports, 5, 14567. https://doi.org/http://dx,doi.org/10.1038/srep14567.

Manero, A., Vilanova, X., Cerdà-Cuéllar, M., \& Blanch, A. R. (2006). Vancomycin-and erythromycinresistant enterococci in a pig farm and its environment. Environmental Microbiology, 8(4), 667674. https://doi.org/10.1111/j.1462-2920.2005.00945.x.

Marshall, B. M., \& Levy, S. B. (2011). Food animals and antimicrobials: impacts on human health. Clinical Microbiology Reviews, 24(4), 718-733. https://doi.org/10.1128/CMR.00002-11.

Matos, A. M., Ornaghi, M. G., Carvalho, V. M., Avila, V. A. D., Bonin, E., Castilho, R. A., Ramos, A. V. G., Baldoqui, D. C., Prado, R. M., \& Prado, I. N. (2021). Atividade antimicrobiana in vitro de uma combinação de óleos vegetais de caju e mamona e de óleos essenciais de cravo, eugenol, timol e vanilina contra bactérias Gram-negaticas e Gram-positivas no rúmen de bovinos. Research, Society and Development, 10(8), 1-15. https://doi.org/10.33448/rsd-v1018.16900

Matthews, C., Crispie, F., Lewis, E., Reid, M., O’Toole, P. W., \& Cotter, P. D. (2019). The rumen microbiome: a crucial consideration when optimising milk and meat production and nitrogen utilisation efficiency. Gut Microbes, 10(2), 115-132. https://doi.org/10.1080/19490976.2018.1505176.

Meschiatti, M. A. P., Gouvêa, V. N., Pellarin, L. A., Batalha, C. D. A., Biehl, M. V, Acedo, T. S., Dórea, 
J. R. R., Tamassia, L. F. M., Owens, F. N., \& Santos, F. A. P. (2019). Feeding the combination of essential oils and exogenous $\alpha$-amylase increases performance and carcass production of finishing beef cattle. Journal of Animal Science, 97(1), 456-471. https://doi.org/10.1093/jas/sky415.

Meyer, N. F., Erickson, G. E., Klopfenstein, T. J., Greenquist, M. A., Luebbe, M. K., Williams, P., \& Engstrom, M. A. (2009). Effect of essential oils, tylosin, and monensin on finishing steer performance, carcass characteristics, liver abscesses, ruminal fermentation, and digestibility. Journal of Animal Science, 87(7), 2346-2354. https://doi.org/10.2527/jas.2008-1493

Monteschio, J. O., Souza, K. A., Vital, A. A. C. P., Guerrero, A., Valero, M. V, Kempinski, E. M. B. C., Barcelos, V. C., Nascimento, K. F., \& Prado, I. N. (2017). Clove and rosemary essential oils and encapsuled active principles (eugenol, thymol and vanillin blend) on meat quality of feedlotfinished heifers. Meat Science, 130, 50-57. https://doi.org/10.1016/j.meatsci.2017.04.002.

Myer, P. R., Smith, T. P. L., Wells, J. E., Kuehn, L. A., \& Freetly, H. C. (2015). Rumen microbiome from steers differing in feed efficiency. PloS One, 10(6), e0129174. https://doi.org/https://doi.org/10.1371/journal.pone.0129174.

Nagaraja, T. G., \& Titgemeyer, E. C. (2007). Ruminal acidosis in beef cattle: The current microbiological and nutritional outlook. Journal of Dairy Science, 90(Supp), E17-E38. https://doi.org/10.3168/jds.2006-478.

Oliveira, C. A., \& Millen, D. D. (2014). Survey of the nutritional recommendations and management practices adopted by feedlot cattle nutritionists in Brazil. Animal Feed Science and Technology, 197, 64-75. https://doi.org/https://doi.org/10.1016/j.anifeedsci.2014.08.010.

Ornaghi, M. G., Passetti, R. A. C., Torrecilhas, J. A., Mottin, C., Vital, A. C. P., Guerrero, A., Sañudo, C., Campo, M. M., \& Prado, I. N. (2017). Essential oils in the diet of young bulls: Effect on animal performance, digestibility, temperament, feeding behaviour and carcass characteristics. Animal Feed Science and Technology, 234, 274-283. https://doi.org/10.1016/j.anifeedsci.2017.10.008.

Patra, A. K. (2010). Meta-analyses of effects of phytochemicals on digestibility and rumen fermentation characteristics associated with methanogenesis. Journal of the Science of Food and Agriculture, 90(15), 2700-2708. https://doi.org/http://dx.doi.org/10.1002/jsfa.4143.

Pinto, A. C. J., \& Millen, D. D. (2018). Nutritional recommendations and management practices adopted by feedlot cattle nutritionists: the 2016 Brazilian survey. Canadian Journal of Animal Science, 99(2), 392-407. https://doi.org/10.1139/cjas-2018-0031.

Rivaroli, D. C. (2013). Níveis de óleos essenciais para bovinos de corte terminados em confinamento com dietaalto grão: desempenho, temperamento animal, características da carcaça e qualidade da carne. In Universidade Estadual Paulista: Vol. Master of (Issue 1). Faculdade de Medicina Veterinária e Zootecnia.

Rivaroli, D. C., Guerrero, A., Valero, M. V., Zawadzki, F., Eiras, C. E., Campo, M. D. M., Sañudo, C., Jorge, A. M., \& Prado, I. N. (2016). Effect of essential oils on meat and fat qualities of crossbred young bulls finished in feedlots. Meat Science, 121, 278-284. https://doi.org/10.1016/j.meatsci.2016.06.017.

Rivaroli, D. C., Ornaghi, M. G., Mottin, C., Prado, R. M., Ramos, T. R., Guerrero, A., Jorge, A. M., \& Prado, I. N. (2017). Essential oils in the diet of crossbred (1/2 Angus vs. $1 / 2$ Nellore) bulls finished in feedlot on animal performance, feed efficiency and carcass characteristics. Journal of Agricultural Science, 9(10), 205-212. https://doi.org/10.5539/jas.v9n10p205-212.

Samuelson, K. L., Hubbert, M. E., Galyean, M. L., \& Löest, C. A. (2016). Nutritional recommendations of feedlot consulting nutritionists: the 2015 New Mexico State and Texas Tech University survey. Journal of Animal Science, 94(6), 2648-2663. https://doi.org/10.2527/jas.2016-0282.

Schäberle, T. F., \& Hack, I. M. (2014). Overcoming the current deadlock in antibiotic research. Trends in Microbiology, 22(4), 165-167. https://doi.org/10.1016/j.tim.2013.12.007.

Souza, K. A., Monteschio, J. O., Mottin, C., Ramos, T. R., Pinto, L. A. M., Eiras, C. E., Guerrero, A., \& Prado, I. N. (2019). Effects of diet supplementation with clove and rosemary essential oils and protected oils (eugenol, thymol and vanillin) on animal performance, carcass characteristics, digestibility, and ingestive behavior activities for Nellore heifers finished in feedl. Livestock Science, 220, 190-195. https://doi.org/10.1016/j.livsci.2018.12.026. 
Torres, R. N. S., Paschoaloto, J. R., Ezequiel, J. M. B., Silva, D. A. V, \& Almeida, M. T. C. (2021). Meta-analysis of the effects of essential oil as an alternative to monensin in diets for beef cattle. The Veterinary Journal, 272, 105659. https://doi.org/10.1016/j.tvj1.2021.105659.

Toseti, L. B., Goulart, R. S., Gouvêa, V. N., Acedo, T. S., Vasconcellos, G. S. F. M., Pires, A. V, Leme, P. R., Netto, A. S., \& Silva, S. L. (2020). Effects of a blend of essential oils and exogenous $\alpha-$ amylase in diets containing different roughage sources for finishing beef cattle. Animal Feed Science and Technology, 269, 114643. https://doi.org/10.1016/j.anifeedsci.2020.114643.

\section{Histórico do artigo:}

Recebido: 2 de agosto de 2021

Aprovado: 2 de setembro de 2021

Disponível online: 9 de novembro de 2021
Licenciamento: Este artigo é publicado na modalidade Acesso Aberto sob a licença Creative Commons Atribuição 4.0 (CC-BY 4.0), a qual permite uso irrestrito, distribuição, reprodução em qualquer meio, desde que o autor e a fonte sejam devidamente creditados. 